\title{
La classe inversée: Impact sur le sentiment d'efficacité personnelle et l'utilisation des résultats probants chez des étudiantes en sciences infirmières (Flipped classroom: impact on self-efficacy and evidence-based practice for nursing students)
}

\author{
Laurence Harvey \\ Université de Montréal, laurence.harvey.2@umontreal.ca \\ Marjolaine Héon \\ Université de Montréal, marjolaine.heon@umontreal.ca \\ Jacinthe I. Pepin \\ Université de Montréal, jacinthe.pepin@umontreal.ca \\ Louise Boyer \\ University of Montreal, louise.boyer@umontreal.ca
}

Follow this and additional works at: https://qane-afi.casn.ca/journal

Part of the Educational Methods Commons, and the Other Nursing Commons

\begin{abstract}
Recommended Citation
Harvey, Laurence; Héon, Marjolaine; Pepin, Jacinthe I.; and Boyer, Louise (2018) "La classe inversée: Impact sur le sentiment d'efficacité personnelle et l'utilisation des résultats probants chez des étudiantes en sciences infirmières (Flipped classroom: impact on self-efficacy and evidence-based practice for nursing students)," Quality Advancement in Nursing Education - Avancées en formation infirmière: Vol. 4: Iss. 1, Article 4.
\end{abstract}

DOI: https://doi.org/10.17483/2368-6669.1136

This Article is brought to you for free and open access by Quality Advancement in Nursing Education - Avancées en formation infirmière. It has been accepted for inclusion in Quality Advancement in Nursing Education - Avancées en formation infirmière by an authorized editor of Quality Advancement in Nursing Education - Avancées en formation infirmière. 


\section{Problématique}

La pratique fondée sur des résultats probants (PFRP) s'impose, dans la profession infirmière et dans bien d'autres, comme une avenue à privilégier afin de favoriser des soins de qualité (Association canadienne des écoles de sciences infirmières [ACÉSI], 2010). La PFRP serait en ce sens un gage de soins efficients et sécuritaires (Christie, Hamill, et Power, 2012; Polit et Beck, 2006; Winters et Echeverri, 2012). Elle est définie comme un processus continu et interactif de prise de décision clinique selon les meilleurs résultats disponibles dans les écrits (Association des infirmières et infirmiers du Canada, 2002). En sciences infirmières, cette approche décisionnelle doit, en plus de recourir aux résultats probants, être basée sur l'expérience personnelle et professionnelle du clinicien, les besoins et préférences de la personne ainsi que les ressources du milieu (DiCenso, Guyatt, et Ciliska, 2005). La PFRP, en complémentarité à une approche caring, permet d'assurer les meilleurs résultats de soins chez les personnes (Melnyk et Fineout-Overholt, 2010), où les connaissances issues de la recherche enrichissent la compréhension de l'expérience de la personne (Faculté des sciences infirmières, 2012). La PFRP permet aussi de potentialiser l'autonomie, la satisfaction et l'épanouissement de l'infirmière (Levin, Fineout-Overholt, Melnyk, Barnes, et Vetter, 2011). Pour ces raisons, les établissements universitaires s'investissent dans l'offre d'une formation infirmière permettant le développement d'habiletés nécessaires à une approche de soins fondée sur des résultats probants (ACÉSI, 2015).

Malgré son importance, la PFRP peine à prendre sa place dans les milieux de soins, alors que les résultats probants ne seraient pas une source d'information privilégiée chez plusieurs infirmières (Melnyk, Fineout-Overholt, Gallagher-Ford, et Kaplan, 2012; Pravikoff, Tanner, et Pierce, 2005). Une étude suédoise indique en ce sens que 44,4\% des infirmières nouvellement diplômées n'auraient jamais recours aux résultats probants dans leur pratique (Forsman, Wallin, Gustavsson, et Rudman, 2012). Une étude américaine rapporte d'ailleurs que seuls 34,5\% des infirmières présenteraient des habiletés liées à la PFRP (Melnyk et al., 2012). Ces habiletés font référence au repérage, à l'analyse et à l'utilisation de résultats probants dans la pratique. La formation initiale ou continue serait l'élément le plus aidant pour l'utilisation des résultats probants dans la pratique (Melnyk et al., 2012). En outre, une étude transversale américaine rapporte que $50 \%$ des infirmières n'auraient pas les habiletés nécessaires à une utilisation efficace des bases de données, ce qui écarterait les résultats probants du processus de décision clinique, au profit des connaissances professionnelles de l'équipe soignante (Pravikoff et al., 2005). Ceci renforce donc l'hypothèse qu'une faible utilisation des résultats probants serait causée, entre autres, par des habiletés limitées dans le processus de repérage et d'évaluation critique de documents scientifiques (Brown, Wickline, Ecoff, et Glaser, 2009; Jones, Crookes, et Johnson, 2011; Melnyk et al., 2012).

Outre les habiletés, les attitudes envers la recherche ont été identifiées comme un facteur déterminant à la PFRP (Squires, Estabrooks, Gustavsson, et Wallin, 2011), ce que des auteurs lient au faible sentiment d'efficacité en la matière (Callister, Matsumura, Lookinland, Mangum, et Loucks, 2005). Une revue systématique indique d'ailleurs qu'une perception négative quant aux habiletés personnelles dans le domaine de la recherche serait une des principales barrières à la PFRP (Kajermo et al., 2010). Cette perception, dans le domaine de la psychologie, fait référence au concept de sentiment d'efficacité personnelle (SEP), sentiment à la base des actions d'une personne (Bandura, 2003). Les infirmières ayant un SEP élevé dans le domaine de la recherche scientifique auraient recours aux résultats probants de 5,5 à 24 fois plus souvent que leurs collègues ayant un SEP faible (Wallin, Bostrom, et Gustavsson, 2012). Le SEP, issu de la théorie sociale 
cognitive (Bandura, 2003), semble donc être un déterminant important de la PFRP, conjointement au développement d'habiletés dans le processus de recherche.

Une faculté des sciences infirmières québécoise a inscrit dans ses programmes de premier cycle universitaire (baccalauréat initial et DEC-BAC) une compétence spécifique se rapportant à la PFRP, intitulée «Traiter toute activité disciplinaire et professionnelle avec rigueur scientifique» (Faculté des sciences infirmières, 2015, p. 24), puisque cette dernière est jugée essentielle à la pratique de tout professionnel de la santé (ACESI, 2015; Finkelmann et Kenner, 2009). Le libellé de cette compétence indique qu'à la fin de la troisième année, une étudiante doit être en mesure de critiquer et d'évaluer la rigueur et la pertinence d'écrits scientifiques, de réaliser une recension des écrits sur une situation rencontrée et d'appuyer sa pratique sur des résultats probants (Faculté des sciences infirmières, 2015).

Les programmes, élaborés selon l'approche par compétences (APC) telle que définie par Lasnier (2000), visent le développement de compétences qui seront déployées dans la pratique. Une compétence est définie comme « un savoir-agir complexe prenant appui sur la mobilisation et la combinaison efficaces d'une variété de ressources internes et externes à l'intérieur d'une famille de situations $\gg$ (Tardif, 2006, p.22).

Malgré l'intégration d'activités d'apprentissage visant le développement de la compétence dans l'ensemble du curriculum de cette faculté, des observations ont permis de cerner un besoin d'apprentissage des étudiantes, particulièrement à la dernière session, où la l'élaboration d'une recension des écrits dans le cadre d'un projet intégrateur, qui vise la réalisation du plein potentiel de pratique d'une infirmière clinicienne auprès d'une clientèle spécifique selon les résultats probants, sollicite particulièrement la compétence de rigueur scientifique.

Afin de contribuer à l'avancement de la formation de premier cycle des infirmières quant à la PFRP, une activité d'apprentissage complémentaire aux activités existantes a été élaborée et mise à l'essai, où elle visait à favoriser un SEP positif en soutien à la compétence de rigueur scientifique. Questions de recherche : 1 - Quel est l'impact d'une activité de classe inversée sur le SEP et l'utilisation des résultats probants ? 2- Quelle est l'appréciation des étudiantes de l'activité de classe inversée ? Le but de cet article est de présenter l'activité d'apprentissage utilisant la méthode de classe inversée ainsi que ses retombées sur le SEP des étudiantes en lien avec l'utilisation des résultats probants dans leurs prises de décisions cliniques.

\section{Cadre de référence}

Le SEP ayant été identifié comme un déterminant de la PFRP, le cadre de référence choisi pour ce projet est la théorie sociale cognitive, développée par Bandura (2003). Cet auteur situe le SEP comme une composante centrale à la base de la motivation et de la persévérance d'une personne. En effet, une personne convaincue d'être en mesure de réussir quelque chose s'y investira de façon importante. Inversement, une personne qui ne se croit pas en mesure de réussir adoptera des attitudes de résignation ou d'apathie. Le SEP n'est toutefois pas tributaire des aptitudes que détient la personne. En effet, une personne qui détient peu d'aptitudes, mais une forte croyance d'efficacité personnelle aura de bonnes chances de réussir une activité, car elle y mettra efforts et persévérance (Bandura).

Le SEP est influencé par quatre éléments distincts (Bandura, 2003). L'expérience de maîtrise, l'élément le plus significatif, fait référence aux expériences personnelles, telles que les échecs, les succès ou toute performance antérieure. Le deuxième élément, l'expérience vicariante, 
correspond à l'observation ou au modelage sur les pairs. En effet, l'échec ou la réussite du pair dans une situation donnée influencera le SEP chez une personne. Le troisième élément, la persuasion verbale, correspond aux encouragements ou rétroactions constructives d'une ou des personnes signifiantes (Bandura). Enfin, l'état physiologique ou émotionnel réfère à l'interprétation d'une personne de ses réactions physiologiques ou émotionnelles afin d'évaluer sa capacité à performer dans une situation donnée (Bandura). Le SEP n'est toutefois pas synonyme de confiance en soi (Bandura). En effet, la confiance en soi est un sentiment plus diffus et général, alors que le SEP s'applique à un élément ou une activité en particulier. Dans le cadre du présent projet, le concept de SEP est interprété comme la croyance, chez les étudiantes, en leurs habiletés à repérer, analyser et utiliser des résultats probants dans leurs activités disciplinaires et professionnelles.

\section{Activités favorisant le SEP}

Le SEP est un facteur prédictif statistiquement significatif de la réussite des étudiantes en sciences infirmières (McLauguin, Moutray, et Muldoon, 2008). En ce sens, il est intéressant d'examiner autant la perception de capacité que la capacité elle-même (Pajares et Urdan, 2006). Une analyse des écrits sur le SEP permet de constater que des activités d'apprentissage ont le potentiel de favoriser un SEP élevé chez des étudiantes. En effet, des stratégies pédagogiques favorisant la participation active auraient de meilleures retombées quant au SEP que des activités plus magistrales. Des activités telles que la simulation (Kameg, Clochesy, Mitchell, et Suresy 2010), l'apprentissage par les pairs, les activités réflexives permettraient de favoriser un SEP positif dans les habiletés cliniques chez les étudiantes (Watt, Murphy, Pascoe, Scanlon, et Gan, 2011). Par ailleurs, des séminaires de formation combinés à des présentations magistrales pourraient augmenter de façon significative le SEP dans l'analyse d'articles scientifiques chez des étudiantes infirmières (Jones et al., 2011). Des expériences de mentorat quant à l'utilisation des résultats probants dans la pratique pourraient favoriser un SEP positif chez les infirmières (Swensen-Brick et Reineck, 2009). En somme, on constate que des activités qui permettent aux étudiantes de s'impliquer de façon active dans leurs apprentissages seraient plus favorables au développement d'un SEP.

\section{Activités favorisant l'utilisation des résultats probants}

Les écrits ont été recensés afin d'identifier les activités d'apprentissage ayant le potentiel de favoriser l'utilisation des résultats probants chez les infirmières. Seuls les écrits mettant de l'avant des activités cohérentes avec l'APC ont été retenus. Un total de six types d'activités ont été identifiés, soit l'apprentissage par projet (Foss, Kvingne, Larsson, et Athlin, 2014; Gray, 2010; Heye et Stevens, 2009; Jakubec et Astle, 2013; Odell et Barta, 2011), le club de lecture (Chouinard et al., 2015; Mattila, Rekola, Koponen, et Eriksson, 2013), l'atelier (Smith-Strom et Norvedt, 2008; Jones, et al., 2011; Zhang, Zeng, Chen, et Li, 2012), le jeu (Miecznikoski Phillips, 2014), l'apprentissage par les pairs (Thomson, Smith, et Annesley, 2014) et la classe inversée (Strickland, Gray, et Hill, 2012). Ces activités, toutes appréciées par les étudiantes, ont le potentiel de développer des habiletés d'analyse et de critique des résultats probants (Heye et Stevens, 2009; Chouinard et al., 2015; Mattila et al., 2013; Smith-Strom et Norvedt, 2008; Zhang et al., 2012), des attitudes positives envers la recherche (Foss et al., 2014; Zhang et al., 2012), un SEP au regard de la PFRP (Jones et al., 2011; Foss et al., 2014), de la motivation (Chouinard et al., 2015; SmithStrom et Norvedt, 2008; Strickland et al., 2012) et des connaissances quant au processus de recherche (Thompson et al.; Strickland et al.). Parmi l'ensemble des activités d'apprentissage 
recensées, la classe inversée a été retenue, considérant le contexte de la mise à l'essai et l'aspect novateur de l'activité. Ce choix est justifié dans la section suivante.

\section{Élaboration de l'activité d'apprentissage}

\section{Choix de l'activité}

Une analyse du milieu a été effectuée afin de déterminer l'activité d'apprentissage ayant le potentiel de s'intégrer aux activités existantes. D'abord, le programme de baccalauréat est d'une durée de trois ans pour les étudiantes ayant une formation collégiale en sciences de la nature (DEC 2 ans) et d'une durée de deux ans pour celle ayant réussi une formation technique en soins infirmiers (DEC 3 ans). L'unité (5 crédits) dans laquelle s'est inscrite l'activité est le cours final du cursus de premier cycle qui est élaboré selon le principe de l'apprentissage par projet. Il est composé de 11 séances où les apprentissages se réalisent en groupes de codéveloppement, composés de 6 à 10 étudiantes. À travers ces séances, les étudiantes sont appelées à développer un projet individuel de recension des écrits sur le plein potentiel de pratique d'une infirmière clinicienne auprès d'une clientèle spécifique rencontrée en stage. Les étudiantes doivent aussi réaliser, en groupe de trois ou quatre, un club de lecture, où elles doivent présenter une évaluation critique de la pertinence, de la rigueur et des possibilités de transferts d'une étude scientifique. Ainsi, des activités d'apprentissage recensées, l'apprentissage par projet, le club de lecture de même que l'atelier (groupes de codéveloppement) sont des stratégies qui étaient déjà mises de l'avant dans le milieu.

Étant donné la nature des travaux demandés aux étudiantes, soit la réalisation d'une recension des écrits sur une clientèle spécifique, de même que des activités déjà mises en place dans le cours, une activité de classe inversée a été choisie. Le sujet spécifique pour l'activité était la réalisation d'une recension des écrits. L'intention pédagogique était d'offrir une activité permettant aux étudiantes de développer leurs habiletés à repérer, critiquer et évaluer la rigueur et la pertinence d'écrits scientifiques, de réaliser une recension des écrits et d'appuyer leur pratique sur des résultats probants.

La classe inversée est définie comme le fait d'offrir le contenu théorique d'un cours avant la période de classe, sous forme audio ou vidéo, afin de pouvoir réaliser des activités permettant la participation active des étudiantes en classe (Roy, 2014). Cette méthode est cohérente avec l'APC, car elle prône l'autonomie de l'étudiante, mais surtout sa participation active lors des présences en classe (Schlairet, Green, et Benton, 2014). Elle comporte divers avantages, dont la possibilité pour les étudiantes d'avoir accès en tout temps au contenu théorique (Roy) et donc de l'écouter au moment choisi et possiblement à plusieurs reprises. Cette caractéristique de l'activité lui permet d'être adaptée à la réalité des étudiantes infirmières (horaires variés) et au rythme de chacune, ce qui n'est pas possible avec une présentation magistrale, à moins qu'elle ne soit enregistrée.

\section{Élaboration}

Un plan a d'abord été élaboré, en fonction du contenu théorique à apprendre pour la réalisation d'une recension des écrits. Ce plan a permis de dégager dix thèmes, où chacun a fait l'objet d'une capsule distincte (Tableau 1). Pour chacune des capsules, un script a été rédigé. La capsule 0 faisait l'objet d'une présentation de la méthode pédagogique. Le contenu des capsules $1,2,3,3.1,3.2,4,5$ et 6 portait sur différentes notions théoriques essentielles à la réalisation d'une recension des écrits. Le cadre de référence a guidé la création de ces huit capsules, alors que celles- 
ci visaient à stimuler les expériences de maitrise ainsi que l'état physiologique ou émotionnel, deux des sentiments à la base du SEP (Bandura, 2003). La capsule 7 contenait la présentation de deux nouveaux diplômés partageant leur expérience de recension des écrits réalisée l'année précédente, dans le même cours. Cette capsule avait pour but d'offrir un moment de partage des expériences vicariantes ainsi que de la persuasion verbale quant à la capacité de réussite des étudiantes dans leur projet.

Pour le montage des capsules, la lecture verbale du script a ensuite été enregistrée, puis le contenu visuel a été élaboré sur la plateforme Powtoon. L'enregistrement audio a ensuite été fusionné au contenu visuel puis exporté en format $\mathrm{mp} 4$. De cette façon, 10 capsules d'une durée variant entre 1 et 11 minutes ont été développées, pour un total de 56 minutes de visionnement.

Tableau 1

Thèmes des capsules audiovisuelles

\begin{tabular}{lll}
\hline Capsules & Thème & Durée \\
\hline Capsule 0 & $\begin{array}{l}\text { Présentation de la classe inversée sur la recension } \\
\text { des écrits }\end{array}$ & 2 minutes 30 secondes \\
Capsule 1 & La recherche infirmière & 6 minutes 32 secondes \\
Capsule 2 & Le repérage des écrits & 3 minutes 53 secondes \\
Capsule 3 & L'analyse d'un article de recherche & 2 minutes 59 secondes \\
Capsule 3.1 & Le devis quantitatif & 8 minutes 38 secondes \\
Capsule 3.2 & Le devis qualitatif & 4 minutes 27 secondes \\
Capsule 4 & L'analyse d'un article de synthèse & 2 minutes 24 secondes \\
Capsule 5 & La recension des écrits & 8 minutes 22 secondes \\
Capsule 6 & Les principales erreurs à éviter dans la rédaction & 5 minutes 12 secondes \\
& d'une recension des écrits & 11 minutes 33 secondes \\
\hline
\end{tabular}

Les capsules ont été déposées sur la plateforme YouTube qui permet une diffusion web en simultané, sans téléchargement (contrairement au format baladodiffusion).

Comme la classe inversée implique aussi une séance d'activité en classe, un atelier a ensuite été préparé. D'une durée de trois heures, celui-ci avait pour but de permettre aux étudiantes de concrétiser leurs apprentissages réalisés à travers les capsules par une période d'échanges et de questions.

\section{Mise à l'essai de l'activité}

Après avoir obtenu l'approbation du comité d'éthique de l'université ciblée (16-026CERES-D) ainsi que du comité de gestion pédagogique du premier cycle de la Faculté des sciences infirmières concernée, les capsules ont été rendues disponibles aux étudiantes par le site du cours, à l'aide d'un lien URL protégé ${ }^{1}$ vers la plateforme YouTube. Les étudiantes ont été avisées de l'importance du visionnement complet (56 minutes) des capsules avant la tenue de l'atelier. Dix jours après la mise en ligne, l'atelier a été animé par deux responsables du projet. Pour cette occasion, les 248 étudiantes ont été séparées en deux groupes de 124 étudiantes. Les capsules sont

\footnotetext{
${ }^{1}$ Seules les personnes ayant le lien URL pouvaient accéder à la vidéo.
} 
demeurées disponibles sur le site du cours jusqu'à la remise des travaux de recension d'écrits par les étudiantes, soit environ 2 mois après la diffusion initiale. Chaque capsule a été visionnée en moyenne 450 fois, ce qui signifie que chaque capsule a été visionnée approximativement deux fois par chaque étudiante. La capsule la plus visionnée est la capsule 1 (667 visionnements) et la moins visionnée est la 3.2 (393 visionnements).

\section{Mesure des retombées}

Les retombées de la mise à l'essai de l'activité sur le SEP quant à la compétence de rigueur scientifique ont été évaluées à l'aide d'un questionnaire élaboré spécifiquement pour ce projet. Le questionnaire, en format papier, a été distribué à trois reprises, soit avant la période de disponibilité des capsules audiovisuelles $\left(T_{1}\right)$, immédiatement après l'atelier en classe $\left(T_{2}\right)$, puis six semaines plus tard, soit une semaine après la remise de la recension des écrits $\left(\mathrm{T}_{3}\right)$. La complétion du questionnaire au $\mathrm{T}_{1}$ permet d'établir une base de référence pour l'analyse des données. Les données recueillies au $\mathrm{T}_{2}$ permettent d'apprécier l'effet de l'activité sur le SEP immédiatement après sa tenue, alors que les données recueillies au $\mathrm{T}_{3}$ permettent d'évaluer si l'effet de l'activité sur le SEP se poursuit dans le temps. Afin de décrire les caractéristiques des étudiantes, une première section du questionnaire a permis de recueillir des données sociodémographiques, telles que l'âge, le sexe, le cheminement d'appartenance, la formation antérieure et le nombre d'années d'expérience clinique en tant qu'infirmière. La deuxième section qui comportait 15 énoncés cherchait à connaître le niveau de SEP et à évaluer les attitudes des étudiantes envers la PFRP, de même que leur intention et leur utilisation des résultats probants. Les énoncés 1 à 11 se sont basés à la fois sur les recommandations de Bandura (2006) quant à la construction d'un outil d'évaluation du SEP et du niveau attendu de développement de la compétence de rigueur scientifique en $3^{\mathrm{e}}$ année selon le référentiel des compétences au baccalauréat (Faculté des sciences infirmières, 2015). Bandura (2003) rappelle l'importance de mesurer la capacité perçue et non l'intention. Cette notion de capacité s'est traduite dans les énoncés par «je me sens capable de ...». Une échelle de type Likert à 5 critères $^{2}$ a été choisie parce qu'elle convient à la mesure d'attributs personnels (Polit et Beck, 2006) et qu'elle est cohérente avec la théorie sociale cognitive (Kennedy, 2013). Ont été ajoutés au questionnaire des énoncés concernant l'utilisation des résultats probants dans la pratique (énoncé 12), l'intention d'y avoir recours dans le futur (énoncé 13) et des attitudes envers la PFRP (énoncés 14 et 15). Afin d'évaluer l'appréciation des étudiantes quant à l'intervention, un ajout de cinq énoncés, soit trois concernant l'appréciation de l'activité (énoncés 16, 18 et 19) et deux concernant l'utilité des activités pour les apprentissages (énoncés 17 et 20), ont été ajoutés au questionnaire distribué au $\mathrm{T}_{2}$. À ce questionnaire ont aussi été ajoutées quatre questions ouvertes afin de recueillir les commentaires des étudiantes quant à leur appréciation de l'activité. Cet ajout au questionnaire du $\mathrm{T}_{2}$ permet d'évaluer l'appréciation et la perception d'utilité de l'activité d'apprentissage, mais n'influence pas l'analyse des données puisque les énoncés 1 à 15 demeurent les mêmes qu'aux $\mathrm{T}_{1}$ et $\mathrm{T}_{3}$.

Les étudiantes ont été informées du caractère libre de la complétion des questionnaires et de la liberté de se désister à tout moment. Les responsables du projet ont quitté la salle de cours à chaque moment, lors de la complétion des questionnaires et les ont récupérés via une enveloppe cachetée. Un rappel a été fait sur l'incapacité de relier les questionnaires aux étudiantes et l'absence d'impact sur les résultats académiques de la non-participation au projet. La recherche ne

\footnotetext{
25 : Tout à fait en accord; 4 : En accord; 3 : Ni en accord, ni en désaccord; 2 : Pas d'accord; 1 : Pas du tout en accord.
} 
s'adressait pas à une population en situation de vulnérabilité; toutes les participantes étaient des étudiantes du cours.

\section{Participantes}

L'activité s'adressait à l'ensemble des étudiantes inscrites au cours de fin de programme $(n=248)$. Le Tableau 2 indique qu'entre $138\left(T_{2}\right)$ et $219\left(T_{1}\right)$ d'entre elles ont participé à l'un des trois temps de mesure de la collecte de données. Il présente aussi les données sociodémographiques des participantes. Elles étaient majoritairement des femmes (variant de 87,7\% à 89,4\% selon le temps de collecte de données), âgées en moyenne de 25 ans, provenant en plus grande proportion du programme de baccalauréat initial (variant de 64,8\% à 70,3\%) nommé « cohorte 1 », que du programme de formation intégrée DEC-BAC nommé «cohorte 2 ». Elles n'avaient, pour la plupart, que la formation requise à l'admission au programme $(79 \%$ à $85,3 \%)$ et en moyenne un an d'expérience en tant qu'infirmières, même si quelques-unes avaient une expérience clinique allant jusqu'à vingt ans.

Tableau 2

Caractéristiques sociodémographiques des participantes

\begin{tabular}{lccc}
\hline Temps & 1 & 2 & 3 \\
$\mathrm{n}$ & 219 & 138 & 199 \\
\hline Age & & & \\
$\quad$ Moyen & 25 & 25 & 25 \\
$\quad$ Minimum & 21 & 21 & 21 \\
Maximum & 43 & 47 & 47 \\
Sexe & & & \\
Féminin & $88,1 \%$ & $87,7 \%$ & $89,4 \%$ \\
Masculin & $11,9 \%$ & $12,3 \%$ & $10,6 \%$ \\
\hline Formation antérieure & & & \\
Baccalauréat initial (cohorte 1) & $64,8 \%$ & $70,3 \%$ & $66,3 \%$ \\
Baccalauréat intégré DECBAC (cohorte 2) & $35,2 \%$ & $29,7 \%$ & $33,7 \%$ \\
DEC sciences ou technique infirmière seulement & $85,3 \%$ & $81,9 \%$ & $79 \%$ \\
Certificat universitaire & $0,5 \%$ & $0,7 \%$ & $1,0 \%$ \\
Baccalauréat autre & $8,7 \%$ & $11,6 \%$ & $9,0 \%$ \\
Formation infirmière hors Québec & $4,6 \%$ & $0,7 \%$ & $9,0 \%$ \\
Formation de 2e cycle & $0,9 \%$ & $5,1 \%$ & $2,0 \%$ \\
\hline Années d'expérience en tant qu'infirmière & & & \\
Moyenne & 1 & 1 & 1 \\
Minimum & 0 & 0 & 0 \\
Maximum & 12 & 12 & 20
\end{tabular}

\section{Analyses statistiques et retombées de la classe inversée}

\section{Retombées sur le SEP, les attitudes et l'utilisation de la recherche}

Avec l'aide d'un statisticien, un test t pour l'égalité des moyennes a été réalisé afin de comparer les différences entre les deux cohortes. Le seuil de signification a été déterminé à $\mathrm{p}<$ 
0,05. Les moyennes du SEP, des attitudes, de l'intention et de l'utilisation des résultats probants aux trois temps de mesure pour les deux cohortes confondues sont présentées au Tableau 3, alors que les moyennes pour les cohortes comparées sont présentées au Tableau 4. L'utilisation d'une échelle de Likert aux trois temps de mesure sans appariement des participants n'a pas permis de déterminer avec certitude l'effet de l'activité sur les étudiantes, même si une progression peut être constatée pour plusieurs énoncés. Précisons tout de même que les moyennes et différences des cohortes prises séparément permettent d'identifier certains éléments distinctifs.

Tableau 3

SEP, attitudes, intentions et utilisation des résultats probants aux trois temps de mesure, cohortes confondues

\section{Énoncés}

1. Je me sens capable de repérer des articles scientifiques pertinents sur les bases de données

2. Je me sens capable de comprendre un article scientifique

3. Je me sens capable de réaliser une évaluation critique des différentes sections d'un article

4. Je me sens capable de réaliser une évaluation critique d'une étude qualitative

5. Je me sens capable de réaliser une évaluation critique d'une étude quantitative

6. Je me sens capable de réaliser une évaluation critique d'une revue des écrits

7. Je me sens capable d'évaluer la possibilité de transfert des résultats d'un article scientifique à ma clientèle spécifique

8. Je me sens capable d'utiliser les résultats d'un article scientifique dans mes travaux scolaires

9. Je me sens capable d'utiliser les résultats d'un article scientifique dans ma pratique professionnelle

10. Je me sens capable de réaliser une recension des écrits

$\begin{array}{cccccc}\mathrm{T} & \mathrm{N} & \mathrm{M} & \mathrm{E} & \text { Min. } & \text { Max. } \\ 1 & 219 & 3,95 & , 67 & 2,0 & 5,0 \\ 2 & 138 & 4,11 & , 54 & 3,0 & 5,0 \\ 3 & 199 & 4,45 & , 58 & 5,0 & 5,0 \\ 1 & 219 & 3,95 & , 60 & 2,0 & 5,0 \\ 2 & 138 & 4,07 & , 55 & 2,0 & 5,0 \\ 3 & 199 & 4,38 & , 56 & 2,0 & 5,0 \\ 1 & 219 & 3,49 & , 72 & 2,0 & 5,0 \\ 2 & 138 & 3,82 & , 61 & 2,0 & 5,0 \\ 3 & 199 & 4,10 & , 66 & 2,0 & 5,0 \\ 1 & 219 & 3,49 & , 72 & 2,0 & 5,0 \\ 2 & 138 & 3,84 & , 65 & 2,0 & 5,0 \\ 3 & 199 & 4,10 & , 59 & 3,0 & 5,0 \\ 1 & 219 & 3,57 & , 71 & 2,0 & 5,0 \\ 2 & 138 & 3,93 & , 66 & 2,0 & 5,0 \\ 3 & 199 & 4,14 & , 65 & 1,0 & 5,0 \\ 1 & 219 & 3,12 & , 81 & 1,0 & 5,0 \\ 2 & 138 & 3,63 & , 73 & 1,0 & 5,0 \\ 3 & 199 & 3,84 & , 75 & 1,0 & 5,0 \\ 1 & 219 & 3,81 & , 64 & 2,0 & 5,0 \\ 2 & 138 & 4,16 & , 63 & 2,0 & 5,0 \\ 3 & 199 & 4,38 & , 57 & 3,0 & 5,0 \\ 1 & 219 & 4,16 & , 50 & 2,0 & 5,0 \\ 2 & 138 & 4,38 & , 54 & 3,0 & 5,0 \\ 3 & 199 & 4,54 & , 54 & 3,0 & 5,0 \\ 1 & 219 & 3,66 & , 75 & 1,0 & 5,0 \\ 2 & 138 & 4,11 & , 64 & 3,0 & 5,0 \\ 3 & 199 & 4,23 & , 66 & 2,0 & 5,0 \\ 1 & 218 & 3,00 & , 88 & 1,0 & 5,0 \\ 2 & 138 & 3,69 & , 70 & 2,0 & 5,0 \\ 3 & 198 & 4,11 & , 73 & 1,0 & 5,0 \\ 1 & 219 & 3,78 & , 64 & 2,0 & 5,0\end{array}$




\begin{tabular}{lcccccc}
\hline \multicolumn{1}{c}{ Énoncés } & $\mathrm{T}$ & $\mathrm{N}$ & $\mathrm{M}$ & $\mathrm{E}_{\mathrm{T}}$ & Min. & Max. \\
\cline { 1 - 5 } 11. Je me sens capable de communiquer les & 2 & 138 & 4,07 &, 54 & 3,0 & 5,0 \\
résultats de mon évaluation critique d'articles & 3 & 198 & 4,29 &, 65 & 2,0 & 5,0 \\
scientifiques à mes pairs & & & & & & \\
12. Dans ma pratique ou en stage, je consulte & 1 & 219 & 3,28 &, 99 & 1,0 & 5,0 \\
régulièrement des écrits scientifiques & 2 & 138 & 3,62 &, 70 & 1,0 & 5,0 \\
& 3 & 199 & 3,86 &, 88 & 1,0 & 5,0 \\
& 1 & 219 & 3,94 &, 80 & 1,0 & 5,0 \\
13. J'ai l'intention de faire appel aux résultats & 2 & 138 & 4,27 &, 68 & 2,0 & 5,0 \\
probants dans ma pratique & 3 & 199 & 4,30 &, 69 & 1,0 & 5,0 \\
& 1 & 219 & 4,48 &, 65 & 2,0 & 5,0 \\
& 2 & 138 & 4,59 &, 57 & 2,0 & 5,0 \\
14. Je pense que la recherche infirmière est & 3 & 199 & 4,57 &, 54 & 3,0 & 5,0 \\
importante pour la pratique & 1 & 219 & 3,12 & 1,06 & 1,0 & 5,0 \\
& 2 & 138 & 3,37 &, 96 & 1,0 & 5,0 \\
15. La lecture et l'analyse d'articles scientifiques & 3 & 199 & 3,52 & 1,07 & 1,0 & 5,0
\end{tabular}

\section{Deux cohortes confondues}

On observe une augmentation des moyennes pour l'ensemble des énoncés du questionnaire, sauf pour l'énoncé 14 (Je pense que la recherche infirmière est importante pour la pratique), où la moyenne reste relativement stable $\left(\mathrm{T}_{1}: 4,48, \mathrm{~T}_{2}: 4,49, \mathrm{~T}_{3}: 4,57\right)$. La moyenne est toutefois déjà élevée au $\mathrm{T}_{1}$.

Tableau 4

SEP, attitudes, intentions et utilisation des résultats probants aux trois temps de mesure, cohortes comparées

\begin{tabular}{|c|c|c|c|c|c|c|c|c|c|c|c|}
\hline \multirow{3}{*}{ Énoncés } & \multirow{3}{*}{$\mathrm{T}$} & \multicolumn{6}{|c|}{ Moyennes } & \multicolumn{2}{|c|}{$\begin{array}{c}\text { Intervalle de } \\
\text { confiance de la } \\
\text { différence à } \\
95 \%\end{array}$} & \multirow[t]{3}{*}{$\mathrm{t}$} & \multirow[t]{3}{*}{ ddl } \\
\hline & & \multicolumn{3}{|c|}{ Cohorte 1} & \multicolumn{3}{|c|}{ Cohorte 2} & Borne & Borne & & \\
\hline & & $\mathrm{M}$ & $\mathrm{E}_{\mathrm{T}}$ & $\mathrm{n}$ & $\mathrm{M}$ & $\mathrm{E}_{\mathrm{T}}$ & $\mathrm{n}$ & & & & \\
\hline \multirow{3}{*}{$\begin{array}{l}\text { 1. Je me sens capable } \\
\text { de repérer des articles } \\
\text { scientifiques pertinents } \\
\text { sur les bases de données }\end{array}$} & 1 & 4,06 & 63 & 142 & 3,77 & ,71 & 77 &, 10 & ,48 & $3,02 *$ & 141 \\
\hline & 2 & 4,13 &, 55 & 97 & 4,05 &, 50 & 41 &,- 11 &, 28 & ,89 & 83 \\
\hline & 3 & 4,58 &, 55 & 132 & 4,19 &, 56 & 67 & ,22 &, 55 & $4,67 *$ & 132 \\
\hline \multirow{3}{*}{$\begin{array}{l}\text { 2. Je me sens capable } \\
\text { de comprendre un } \\
\text { article scientifique }\end{array}$} & 1 & 4,01 &, 55 & 142 & 3,84 & 67 & 77 &,- 01 &, 34 & 1,91 & 131 \\
\hline & 2 & 4,06 &, 56 & 97 & 4,10 &, 54 & 41 &,- 24 & , 17 &,- 35 & 78 \\
\hline & 3 & 4,47 &, 52 & 132 & 4,19 & ,61 & 67 &, 10 & ,44 & $3,17 *$ & 115 \\
\hline \multirow{4}{*}{$\begin{array}{l}\text { 3. Je me sens capable } \\
\text { de réaliser une } \\
\text { évaluation critique des } \\
\text { différentes sections } \\
\text { d'un article }\end{array}$} & 1 & 3,54 &, 71 & 142 & 3,41 &, 73 & 77 &,- 08 &, 32 & 1,17 & 152 \\
\hline & 2 & 3,81 & ,60 & 97 & 3,83 & ,63 & 41 &,- 26 &, 22 &,- 13 & 72 \\
\hline & 3 & 4,67 & ,66 & 132 & 3,97 & 65 & 67 &, 00 & ,39 & $2,01 *$ & 134 \\
\hline & 1 & 3,47 &, 75 & 142 & 3,53 & ,66 & 77 &,- 25 &, 13 &,- 62 & 173 \\
\hline
\end{tabular}




\begin{tabular}{|c|c|c|c|c|c|c|c|c|c|c|c|}
\hline \multirow{3}{*}{ Énoncés } & \multirow{3}{*}{$\mathrm{T}$} & \multicolumn{6}{|c|}{ Moyennes } & \multicolumn{2}{|c|}{$\begin{array}{l}\text { Intervalle de } \\
\text { confiance de la } \\
\text { différence à } \\
95 \%\end{array}$} & \multirow[t]{3}{*}{ 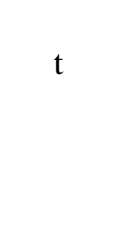 } & \multirow[t]{3}{*}{ ddl } \\
\hline & & \multicolumn{3}{|c|}{ Cohorte 1} & \multicolumn{3}{|c|}{ Cohorte 2} & \multirow{2}{*}{$\begin{array}{l}\text { Borne } \\
\text { Inf. }\end{array}$} & \multirow{2}{*}{$\begin{array}{l}\text { Borne } \\
\text { Sup. }\end{array}$} & & \\
\hline & & $\mathrm{M}$ & $\mathrm{E}_{\mathrm{T}}$ & $\mathrm{n}$ & $\mathrm{M}$ & $\mathrm{E}_{\mathrm{T}}$ & $\mathrm{n}$ & & & & \\
\hline \multirow{5}{*}{$\begin{array}{l}\text { 4. Je me sens capable } \\
\text { de réaliser une } \\
\text { évaluation critique } \\
\text { d'une étude qualitative } \\
\text { 5. Je me sens capable } \\
\text { de réaliser une } \\
\text { évaluation critique } \\
\text { d'une étude quantitative }\end{array}$} & 2 & 3,85 & ,67 & 97 & 3,83 & ,63 & 41 &,- 22 &, 25 &, 14 & 80 \\
\hline & 3 & 4,12 & 61 & 132 & 4,06 &, 55 & 67 &,- 11 & ,23 &, 72 & 145 \\
\hline & 1 & 3,63 & ,69 & 142 & 3,46 & ,08 & 77 &,- 02 &, 38 & 1,76 & 148 \\
\hline & 2 & 3,94 & ,67 & 97 & 3,90 & ,62 & 41 &,- 20 &, 27 &, 30 & 81 \\
\hline & 3 & 4,25 & ,61 & 131 & 3,93 & ,68 & 67 &, 13 &, 52 & $3,30 *$ & 121 \\
\hline \multirow{3}{*}{$\begin{array}{l}\text { 6. Je me sens capable } \\
\text { de réaliser une } \\
\text { évaluation critique } \\
\text { d'une revue des écrits }\end{array}$} & 1 & 3,13 & ,84 & 142 & 3,12 &, 76 & 77 &,- 21 &, 23 & ,09 & 170 \\
\hline & 2 & 3,63 & ,66 & 97 & 3,63 &, 75 & 41 &,- 26 &, 25 &,- 04 & 85 \\
\hline & 3 & 3,86 & ,78 & 132 & 3,82 & 69 & 67 &,- 18 &, 25 & ,32 & 48 \\
\hline \multirow{3}{*}{$\begin{array}{l}\text { 7. Je me sens capable } \\
\text { d'évaluer la possibilité } \\
\text { de transfert des résultats } \\
\text { d'un article scientifique } \\
\text { à ma clientèle } \\
\text { spécifique }\end{array}$} & 1 & 3,83 & ,61 & 142 & 3,78 &, 70 & 77 &,- 13 &, 23 &, 55 & 138 \\
\hline & 2 & 4,16 & 64 & 97 & 4,17 & 63 & 41 &,- 25 &, 22 &,- 14 & 76 \\
\hline & 3 & 4,42 & ,58 & 132 & 4,30 &, 55 & 67 &,- 04 & ,29 & 1,49 & 139 \\
\hline \multirow{3}{*}{$\begin{array}{l}\text { 8. Je me sens capable } \\
\text { d'utiliser les résultats } \\
\text { d'un article scientifique } \\
\text { dans mes travaux } \\
\text { scolaires }\end{array}$} & 1 & 4,19 &, 50 & 142 & 4,10 &, 50 & 77 &,- 05 &, 23 & 1,21 & 157 \\
\hline & 2 & 4,37 & ,57 & 97 & 4,39 & ,49 & 41 &,- 21 &, 17 &,- 20 & 86 \\
\hline & 3 & 4,64 & ,51 & 132 & 4,33 &, 53 & 67 &, 15 & ,47 & $4,00 *$ & 128 \\
\hline \multirow{6}{*}{$\begin{array}{l}\text { 9. Je me sens capable } \\
\text { d'utiliser les résultats } \\
\text { d'un article scientifique } \\
\text { dans ma pratique } \\
\text { professionnelle } \\
\text { 10. Je me sens capable } \\
\text { de réaliser une } \\
\text { recension des écrits }\end{array}$} & 1 & 3,73 & ,70 & 142 & 3,53 & ,82 & &,- 03 & ,41 & 1,76 & 136 \\
\hline & 2 & 4,12 & 63 & 97 & 4,07 & ,65 & 41 &,- 19 & ,29 &, 42 & 74 \\
\hline & 3 & 4,35 & 63 & 132 & 4,0 & ,67 & 67 &, 15 &, 54 & $3,52 *$ & 125 \\
\hline & 1 & 3,09 & ,87 & 141 & 2,83 & ,88 & 77 & ,02 &, 51 & $2,10 *$ & 155 \\
\hline & 2 & 3,75 &, 75 & 97 & 3,54 &, 55 & 41 &,- 01 & ,44 & 1,88 & 101 \\
\hline & 3 & 4,19 & ,67 & 131 & 3,96 & ,81 & &, 01 & ,46 & $2,06^{*}$ & 114 \\
\hline \multirow{3}{*}{$\begin{array}{l}\text { 11. Je me sens capable } \\
\text { de communiquer les } \\
\text { résultats de mon } \\
\text { évaluation critique } \\
\text { d'articles scientifiques à } \\
\text { mes pairs }\end{array}$} & 1 & 3,75 & 69 & 142 & 3,82 &, 56 & 77 &,- 24 &, 10 &,- 76 & 185 \\
\hline & 2 & 4,05 &, 57 & 97 & 4,12 & ,46 & 41 &,- 25 &, 11 &,- 77 & 92 \\
\hline & 3 & 4,37 & ,61 & 131 & 4,14 & ,78 & 67 &, 02 & ,42 & $2,15^{*}$ & 118 \\
\hline \multirow{3}{*}{$\begin{array}{l}\text { 12. Dans ma pratique } \\
\text { ou en stage, je consulte } \\
\text { régulièrement des écrits } \\
\text { scientifiques }\end{array}$} & 1 & 3,46 & ,88 & 142 & 2,95 & 1,09 & 77 & ,22 & ,80 & $3,53 *$ & 131 \\
\hline & 2 & 3,68 & 0,7 & 97 & 3,48 & ,68 & 41 &,- 06 & ,44 & 1,51 & 78 \\
\hline & 3 & 3,95 & ,82 & 132 & 3,67 & ,98 & 67 &, 01 &, 56 & $2,04 *$ & 114 \\
\hline \multirow{3}{*}{$\begin{array}{l}\text { 13. J'ai l'intention de } \\
\text { faire appel aux résultats } \\
\text { probants dans ma } \\
\text { pratique }\end{array}$} & 1 & 4,11 & ,62 & 142 & 3,64 & ,99 & 77 & ,22 &, 71 & $3,80 *$ & 109 \\
\hline & 2 & 4,38 & ,62 & 97 & 4,0 & ,74 & 41 &, 12 & ,64 & $2,89 *$ & 64 \\
\hline & 3 & 4,44 & 61 & 132 & 4,0 &, 76 & 67 & ,24 & ,66 & $4,19 *$ & 110 \\
\hline
\end{tabular}




\begin{tabular}{|c|c|c|c|c|c|c|c|c|c|c|c|}
\hline \multirow{3}{*}{ Énoncés } & \multirow{3}{*}{$\mathrm{T}$} & \multicolumn{6}{|c|}{ Moyennes } & \multicolumn{2}{|c|}{$\begin{array}{c}\text { Intervalle de } \\
\text { confiance de la } \\
\text { différence à } \\
95 \%\end{array}$} & \multirow[t]{3}{*}{$\mathrm{t}$} & \multirow[t]{3}{*}{$\mathrm{ddl}$} \\
\hline & & \multicolumn{3}{|c|}{ Cohorte 1} & \multicolumn{3}{|c|}{ Cohorte 2} & Borne & Borne & & \\
\hline & & $\mathrm{M}$ & $\mathrm{E}_{\mathrm{T}}$ & $\mathrm{n}$ & $\mathrm{M}$ & $\mathrm{E}_{\mathrm{T}}$ & $\mathrm{n}$ & & & & \\
\hline \multirow{3}{*}{$\begin{array}{l}\text { 14. Je pense que la } \\
\text { recherche infirmière est } \\
\text { importante pour la } \\
\text { pratique }\end{array}$} & 1 & 4,61 &, 54 & 142 & 4,23 &, 76 & 77 & ,18 &, 57 & $3,87^{*}$ & 119 \\
\hline & 2 & 4,64 &, 56 & 97 & 4,49 & ,60 & 41 &,- 07 & ,37 & 1,39 & 71 \\
\hline & 3 & 4,70 & ,48 & 132 & 4,33 &, 59 & 67 & ,20 &, 53 & $4,44^{*}$ & 111 \\
\hline \multirow{3}{*}{$\begin{array}{l}\text { 15. La lecture et } \\
\text { l'analyse d'articles } \\
\text { scientifiques génèrent } \\
\text { chez moi de } \\
\text { l'enthousiasme }\end{array}$} & 1 & 3,24 & ,97 & 142 & 2,90 & 1,18 & 77 & ,03 & ,65 & $2,19 *$ & 132 \\
\hline & 2 & 3,43 & ,80 & 97 & 3,22 & 1,26 & 41 &,- 21 & ,63 & 1,01 & 54 \\
\hline & 3 & 3,70 & ,95 & 132 & 3,15 & 1,20 & 67 &, 22 & ,89 & $3,31 *$ & 109 \\
\hline
\end{tabular}

\section{Deux cohortes comparées}

Lorsqu'on compare les deux cohortes, on remarque qu'au $\mathrm{T}_{1}$, les moyennes de la cohorte 1 sont systématiquement plus élevées que celles de la cohorte 2, pour l'ensemble des énoncés. Les tests statistiques indiquent que ces différences sont significatives pour six énoncés $(1,10,12,13$, 14 et 15). Au $\mathrm{T}_{2}$, on observe un tamponnement des différences, expliqué par une augmentation des moyennes pour la cohorte 2. Une seule différence entre les moyennes est significative, pour l'énoncé 13 (intention de faire appel aux résultats probants dans la pratique). $A u \mathrm{~T}_{3}$, on observe un retour des différences entre les cohortes, ce qui s'exprime par une augmentation des moyennes de la cohorte 1. Les différences sont significatives pour l'ensemble des énoncés sauf pour les énoncés 4,6 et 7 .

\section{Cohorte 1 - Programme de baccalauréat initial}

Au Tableau 4, il est possible d'observer une augmentation progressive des moyennes, du $\mathrm{T}_{1}$ au $\mathrm{T}_{3}$, chez les étudiantes de la cohorte 1 pour l'ensemble des énoncés. Puisque les questionnaires n'ont pas été appariés aux étudiantes, il n'est pas possible de déterminer si l'augmentation est statistiquement significative. La moyenne la plus élevée au $\mathrm{T}_{1}$ est à l'énoncé 14 (Je pense que la recherche infirmière est importante pour la pratique), alors que la plus basse est à l'énoncé 10 (Je me sens capable de réaliser une recension des écrits). Au $\mathrm{T}_{2}$, la moyenne la plus élevée est toujours à l'énoncé 14, alors que la moyenne la plus basse est pour l'énoncé 15 ( $L a$ lecture et l'analyse d'articles scientifiques génèrent chez moi de l'enthousiasme). Au $\mathrm{T}_{3}$, la moyenne la plus haute revient à l'énoncé 14 alors que la plus basse reste à l'énoncé 15 .

\section{Cohorte 2 - Programme de baccalauréat intégré DECBAC}

On observe aussi chez les étudiantes de la cohorte 2 une progression générale des moyennes du $\mathrm{T}_{1}$ au $\mathrm{T}_{3}$. Des exceptions s'appliquent toutefois, soit pour les énoncés 8 (Je me sens capable d'utiliser les résultats d'un article scientifique dans mes travaux scolaires), 9 (Je me sens capable d'utiliser les résultats d'un article scientifique dans ma pratique professionnelle), 14 (Je pense que la recherche infirmière est importante pour la pratique) et 15 (La lecture et l'analyse d'articles scientifiques génèrent chez moi de l'enthousiasme), où on observe une diminution des moyennes entre les $\mathrm{T}_{2}$ et $\mathrm{T}_{3}$. 
Il est toutefois à noter que les moyennes de la cohorte 2 sont initialement plus basses ou équivalentes aux moyennes de la cohorte 1 pour la plupart des énoncés. Toujours chez la cohorte 2, la moyenne la plus élevée au $\mathrm{T}_{1}$ est à l'énoncé 14 (Je pense que la recherche infirmière est importante pour la pratique), alors que la plus basse est à l'énoncé 10 (Je me sens capable de réaliser une recension des écrits). Au $\mathrm{T}_{2}$, la moyenne la plus haute est toujours à l'énoncé 14, alors que la plus basse est à l'énoncé 15 (La lecture et l'analyse d'articles scientifiques génèrent chez moi de l'enthousiasme). Au $\mathrm{T}_{3}$, l'énoncé 14 a toujours la moyenne la plus haute, alors que l'énoncé 15 conserve la moyenne la plus basse.

\section{Appréciation de l'activité}

Les énoncés 16 à 20, ajoutés au deuxième questionnaire, permettent de quantifier l'appréciation générale de l'activité par les étudiantes (Tableau 5). Un test t pour égalité des moyennes a aussi été réalisé afin de comparer les deux cohortes. Aucune différence n'est significative. La moyenne est supérieure à 4.0 (En accord) pour l'ensemble des énoncés.

Tableau 5

Appréciation de l'activité de classe inversée

\begin{tabular}{|c|c|c|c|c|c|c|c|c|c|c|}
\hline \multirow[t]{3}{*}{ Énoncés } & \multicolumn{6}{|c|}{ Moyennes } & \multicolumn{2}{|c|}{$\begin{array}{c}\text { Intervalle de } \\
\text { confiance de la } \\
\text { différence à } 95 \%\end{array}$} & \multirow[t]{3}{*}{$\mathrm{t}$} & \multirow[t]{3}{*}{ ddl } \\
\hline & \multicolumn{3}{|c|}{ Cohorte 1} & \multicolumn{3}{|c|}{ Cohorte 2} & Inf & Sup & & \\
\hline & $\mathrm{M}$ & $\mathrm{E}_{\mathrm{T}}$ & $\mathrm{n}$ & $\mathrm{M}$ & $\mathrm{E}_{\mathrm{T}}$ & $\mathrm{n}$ & & & & \\
\hline $\begin{array}{l}\text { 16. J'ai apprécié l'utilisation de } \\
\text { capsules vidéo afin de } \\
\text { m'approprier le contenu théorique }\end{array}$ & 4,80 & ,45 & 96 & 4,78 &, 42 & 41 &,- 14 &, 18 &, 27 & 81 \\
\hline $\begin{array}{l}\text { 17. Le contenu des capsules vidéo } \\
\text { m'a été utile }\end{array}$ & 4,78 & ,44 & 96 & 4,81 &, 40 & 41 &,- 18 &, 13 &,- 30 & 83 \\
\hline $\begin{array}{l}\text { 18. J'ai apprécié le fait de pouvoir } \\
\text { écouter le contenu théorique à la } \\
\text { maison }\end{array}$ & 4,88 &, 32 & 95 & 4,88 &, 33 & 41 &,- 12 & ,13 &, 10 & 73 \\
\hline $\begin{array}{l}\text { 19. J'ai apprécié les activités } \\
\text { réalisées en classe, lors de l'atelier } \\
\text { sur la recension des écrits }\end{array}$ & 4,46 & ,72 & 97 & 4,39 &, 54 & 41 &,- 14 &, 30 & ,66 & 99 \\
\hline $\begin{array}{l}\text { 20. Les activités réalisées en classe, } \\
\text { lors de l'atelier sur la recension des } \\
\text { écrits, m'ont été utiles }\end{array}$ & 4,50 & ,68 & 97 & 4,29 & ,64 & 41 &,- 03 &, 45 & 1,7 & 179 \\
\hline
\end{tabular}

Des questions à réponses ouvertes ajoutées au deuxième questionnaire ont permis de recueillir les commentaires des étudiantes. Des 138 ayant participé au deuxième moment de la collecte de données, 85 ont offert des commentaires.

Questionnées à savoir ce qu'elles ont le plus apprécié de l'activité, des étudiantes ont identifié la clarté des informations et des explications transmises par les capsules $(\mathrm{n}=17)$, l'accessibilité du contenu théorique à la maison $(n=10)$, la durée $(n=5)$ ainsi que le contenu, qui faisait appel aux acquis antérieurs $(n=4)$. Par rapport à l'atelier en classe, des étudiantes rapportent avoir apprécié pouvoir poser leurs questions à tout moment $(n=2)$ de même que d'avoir pu se préparer à l'atelier par le visionnement des capsules $(\mathrm{n}=2)$.

Questionnées à savoir ce qu'elles ont le moins apprécié de l'activité, les étudiantes ont identifié un nombre trop important de questions individuelles très spécifiques au travail de 
recension des écrits $(\mathrm{n}=19)$, reflétant le manque de préparation de certaines étudiantes pour la classe inversée. Certaines ont trouvé l'activité en classe trop longue $(n=8)$. Des étudiantes auraient préféré retrouver des explications plus spécifiques sur les différents niveaux de preuves dans les capsules $(\mathrm{n}=2)$.

Des étudiantes ont proposé des pistes d'amélioration, telles que d'offrir l'activité plus tôt dans le cursus universitaire $(n=30)$ et d'offrir un résumé écrit du contenu des capsules $(n=3)$. Enfin, une étudiante suggère d'offrir un plan de l'activité en classe afin de mieux s'y préparer.

\section{Discussion}

\section{SEP, attitudes, intentions et utilisation des résultats probants}

L'augmentation progressive des moyennes entre les $\mathrm{T}_{1}$ et $\mathrm{T}_{3}$ pour l'ensemble des étudiantes semble indiquer une amélioration du SEP, des intentions et des attitudes quant à la PFRP. Il est toutefois impossible de confirmer que cette augmentation est le fruit de l'activité de classe inversée, puisque les questionnaires n'ont pas été appariés. L'effet Hawthorne, de même l'effet de nouveauté, peuvent avoir influencé à la hausse les moyennes. Les résultats répondent donc ici que partiellement à la première question de recherche. Il est toutefois possible de retirer quelques informations de l'observation de la variation des moyennes.

Les moyennes chez les deux cohortes pour les énoncés 14 et 15, qui concernent les attitudes des étudiantes quant à la PFRP, indiquent que malgré la mise à l'essai de la classe inversée, le recours aux résultats probants ne galvanise pas les étudiantes. Elles reconnaissent toutefois l'importance de ceux-ci pour la qualité de la pratique. Ceci diffère des écrits de Christie et al. (2012), Meeker, Jones, et Flanagan (2008), Pravikoff et al. (2005), et Wallen et al. (2010) qui rapportaient que les étudiantes avaient tendance à sous-estimer l'utilité des résultats probants pour la pratique.

Les différences significatives entre les moyennes des deux cohortes pourraient s'expliquer par trois facteurs : la formation antérieure, le cheminement dans le milieu universitaire et l'expérience dans le milieu clinique. D'une part, la cohorte 1 a reçu, avant sa formation au baccalauréat, une formation collégiale en sciences de la nature, formation qui permet le développement d'habiletés scientifiques, telles que les mathématiques, la biologie, la physique et la chimie. Ce cheminement pourrait favoriser le contact avec les données issues de la recherche et pourrait donc soutenir le développement d'habiletés à chercher, comprendre et analyser des articles de recherche. Ceci pourrait particulièrement expliquer l'existence d'une différence significative dans les attitudes envers la PFRP (énoncés 14 et 15).

D'autre part, au moment de réaliser l'activité, les étudiantes de la cohorte 1 en sont à leur $6^{\mathrm{e}}$ session universitaire, ce qui fait qu'elles ont été exposées depuis plus longtemps à des activités reliées à la PFRP où la compétence de rigueur scientifique a été particulièrement exploitée. Les étudiantes de la cohorte 2 en sont à leur $4^{\mathrm{e}}$ session et ont donc moins été exposées à des activités de PFRP en milieu universitaire. Ceci pourrait aussi expliquer les différences systématiques entre les deux cohortes au $\mathrm{T}_{1}$, qui persistent au $\mathrm{T}_{3}$.

Par ailleurs, les étudiantes de la cohorte 2 étaient déjà infirmières et pratiquent donc déjà, pour la plupart, dans un milieu de soin. Elles seraient donc au fait des différences complexes entre une pratique de soin idéale, fondée sur des résultats probants, et la réalité de pratique, où les ressources limitées des milieux cliniques ne facilitent pas toujours une PFRP. Plus précisément, ceci pourrait expliquer les différences significatives aux énoncés qui concernent les intentions et 
l'utilisation des résultats probants (12 et 13). De plus, les étudiantes de la cohorte 1, n'ayant, dans le meilleur des cas, une expérience de quelques mois, ont pu répondre à l'énoncé 12 en s'inspirant, entre autres, de leurs expériences en stages, où la recherche d'écrits scientifiques y est obligatoire. Ceci pourrait donc influencer à la hausse la moyenne chez cette cohorte.

Enfin, bien qu'il ne soit pas possible de le déterminer avec certitude, l'augmentation des moyennes entre le $\mathrm{T}_{1}$ et le $\mathrm{T}_{3}$ pour les deux cohortes nous permet de supposer que l'activité de classe inversée, en complémentarité des activités existantes et en réponse à un besoin d'apprentissage, a pu favoriser une progression du SEP, des attitudes et du recours à la PFRP.

\section{Appréciation de l'activité}

La moyenne des énoncés 16 à 20 pour les deux cohortes est supérieure à 4,0 (En accord), ce qui permet de supposer une appréciation générale positive de l'activité d'apprentissage et répond donc à la deuxième question de recherche. En ce sens, le contenu de la classe inversée a été utile dans la réalisation du travail de recension des écrits. Dans le même ordre d'idée, la clarté du contenu présenté dans les capsules, de même que la courte durée de chacune d'entre elles semblent avoir été une force.

Le fait que les étudiantes aient exprimé le désir de pouvoir avoir accès plus tôt dans le programme au contenu théorique présenté semble indiquer une expérience d'intégration des ressources et donc un développement de la compétence de rigueur scientifique. En effet, ces témoignages suggèrent que le contenu théorique intégré aux capsules vidéo ainsi que l'atelier a permis une expérience d'itération des ressources développées pendant les dernières années du cursus. Cette occasion d'itération aurait permis aux étudiantes de mobiliser efficacement leurs ressources internes afin de réaliser une réelle expérience d'intégration des ressources nécessaires à la compétence de rigueur scientifique.

Les commentaires quant à la pertinence des questions posées en classe de même que la durée de l'activité peuvent s'expliquer par un trop grand nombre d'étudiantes dans la classe $(n=124)$, ce qui n'aurait pas été favorable aux discussions. Par ailleurs, le nombre d'enseignantes $(\mathrm{n}=2)$ semble avoir été insuffisant pour guider adéquatement les discussions en petits groupes. Toutefois, il semble que l'activité ait tout de même été favorable pour les étudiantes, car une comparaison informelle du nombre de questions sur le forum et par courriel avec les cohortes antérieures indique une nette diminution pour l'année en cours. Ainsi, l'activité a été bénéfique pour l'apprentissage des étudiantes, mais que son organisation doit être revue afin de maximiser les discussions en classe.

\section{Forces du projet}

La principale force du projet réside dans le fait qu'il ait été développé à partir de la théorie sociale cognitive de Bandura (2003). En ce sens, les capsules audiovisuelles ont été élaborées dans un souci de répondre au développement d'un SEP positif dans le domaine de la recherche. Le projet a aussi été développé en étroite collaboration avec la responsable de l'unité afin de répondre à un besoin d'apprentissage. L'appréciation de la part des étudiantes et de la responsable de l'activité, de même que les résultats favorables quant au SEP, nous permettent de déduire que le projet a su répondre, en partie, au besoin d'apprentissage. Ainsi, il est possible de conclure à une bonne acceptabilité du projet. En effet, l'activité de classe inversée a été conservée par la responsable de l'unité d'apprentissage afin d'en assurer la pérennité. L'intégration du projet à travers les activités déjà en place dans le cours est aussi une force. En effet, en s'inspirant de 
l'APC, des besoins d'apprentissage exprimés ainsi que de la structure déjà en place, l'activité a été cohérente et s'est intégrée de manière naturelle au cours.

Enfin, l'une des forces de ce projet est son caractère novateur, alors que le recours à la stratégie était une première. Malgré cela, l'activité a été appréciée, de par son contenu comme sa forme, et a été implantée sans aucun problème technique. Ainsi, il est possible de conclure à une bonne faisabilité du projet.

\section{Limites du projet}

La principale limite de ce projet, puisqu'il s'agissait d'un projet-pilote, est qu'il s'est déroulé dans un seul milieu universitaire, auprès d'un seul groupe d'étudiantes. Il serait en ce sens pertinent d'explorer l'efficacité d'une activité de classe inversée dans un milieu universitaire différent, auprès de plusieurs cohortes. En outre, il aurait été intéressant d'approfondir la collecte de données vers l'expérience vécue des étudiantes à travers la mise à l'essai de l'activité d'apprentissage. Le questionnaire utilisé n'a d'ailleurs pas été validé. De plus, le fait de n'avoir pu appareiller les questionnaires aux étudiantes entre les trois moments de collecte a affaibli la qualité statistique des résultats obtenus et limite la capacité à évaluer l'effet réel de la classe inversée pour le SEP, les attitudes et l'utilisation des résultats probants.

\section{Recommandations pour la formation}

Le recours à la plateforme de diffusion vidéo YouTube dans un contexte d'apprentissage de la recension des écrits pour la pratique, stratégie novatrice en sciences infirmières, s'est avéré une expérience positive. Bien que les écrits recensés aient fait appel à la baladodiffusion pour l'implantation d'une classe inversée (Strickland et al., 2012), l'utilisation de la diffusion web a été retenue pour le présent projet, notamment par sa facilité d'utilisation et l'avantage de ne nécessiter aucun téléchargement donc aucun espace de stockage sur les différents appareils des étudiantes. Puisqu'aucun inconvénient ou problème technique n'a été soulevé et que les étudiantes rapportent avoir apprécié cette méthode, il serait intéressant de se pencher sur les possibilités offertes par les plateformes de diffusion gratuite telles que YouTube dans un contexte d'apprentissage. Dans le même ordre d'idée, l'appréciation des étudiantes quant à la méthode était palpable, particulièrement en ce qui concernait à l'accès au contenu théorique en tout temps. L'utilisation de capsules audiovisuelles semble donc être une avenue intéressante, quoique complémentaire, afin d'offrir un soutien aux étudiantes dans la réalisation de leurs travaux personnels.

L'atelier en classe doit pouvoir favoriser la discussion et la participation active des étudiantes, mais aussi un soutien constant des formateurs. C'est pourquoi il serait préférable de diminuer le nombre d'étudiantes du groupe en classe, ce qui pourrait favoriser la discussion et l'accompagnement. Par ailleurs, puisque le bon fonctionnement de l'atelier est tributaire de la préparation des étudiantes avant la classe, il serait utile de réfléchir à une stratégie qui pourrait potentialiser le nombre d'étudiantes qui visionneront les capsules. En ce sens, la création de questionnaires sommatifs en ligne sur le contenu des capsules pourrait être une avenue intéressante, ce qui pourrait motiver les étudiantes à réaliser le visionnement de l'ensemble des capsules.

Puisque les étudiantes ont exprimé le désir d'avoir accès à du contenu théorique en ligne sur la PFRP plus tôt dans leur cheminement, il serait intéressant d'explorer les besoins d'apprentissage quant à la PFPR en première et deuxième année du programme de baccalauréat. En effet, peut-être que la création de capsules audiovisuelles adaptées aux différents niveaux 
pourrait être bénéfique et permettrait d'assurer une expérience de recherche positive dès la première année de formation.

Enfin, comme un écart semble subsister entre les étudiantes de la cohorte 1 et celles de la cohorte 2, il serait pertinent de se pencher sur des stratégies qui permettraient aux étudiantes issues du programme de formation intégrée DECBAC d'initier le plus rapidement possible un contact positif avec les écrits scientifiques.

\section{Orientations pour la recherche}

Malgré l'observation d'une progression du SEP quant à l'utilisation des résultats probants chez les étudiantes, il n'a pas été possible de déterminer avec certitude si cette progression a été le fruit de l'activité de classe inversée, étant donné les limites de la méthode de collecte et d'analyse des données. Il serait pertinent de recourir à un devis plus rigoureux, soit une étude expérimentale utilisant une approche mixte afin de quantifier l'effet de l'activité sur le SEP, les attitudes et l'utilisation des résultats probants, mais aussi d'explorer en profondeur l'expérience vécue par les étudiantes dans une activité de classe inversée. Par ailleurs, il serait intéressant d'étudier les avantages de l'utilisation de différents outils technologiques (YouTube, Powtoon) dans un contexte universitaire.

\section{Conclusion}

Les étudiantes infirmières doivent aujourd'hui être en mesure d'intégrer les résultats probants dans leurs prises de décisions cliniques. L'activité proposée visait le développement d'un SEP et attitudes favorables à l'utilisation de résultats probants, en soutien au développement de la compétence de rigueur scientifique. Malgré le caractère complexe de la problématique, l'activité de classe inversée, conjointement aux activités du milieu, a su répondre aux besoins d'apprentissage en favorisant la progression du SEP, des attitudes et de l'utilisation des résultats probants chez les étudiantes. La cohérence de l'activité avec le contexte d'apprentissage (APC) a permis une intégration des plus naturelles au milieu. Grâce au caractère novateur des plateformes technologiques utilisées, une appréciation élevée des étudiantes permet de constater que les différentes TIC peuvent offrir des avantages intéressants dans un contexte universitaire. Enfin, la classe inversée est une activité d'apprentissage prometteuse dont les effets à long terme sur le SEP quant à l'utilisation des résultats probants dans la pratique mériteraient d'être plus amplement étudiés. 


\section{Références}

Association canadienne des écoles de sciences infirmières [ACÉSI]. (2010). Plaidoyer pour des Canadiens en meilleure santé: Formation en sciences infirmières pour le XXIe siècle. Ottawa, ON : Auteur.

ACÉSI. (2015). Cadre national de l'ACÉSI sur la formation infirmière. Ottawa, ON : Auteur.

Association des infirmières et infirmiers du Canada. (2002). Prise de décision et pratique infirmière éclairées par des preuves. Ottawa, ON : Auteur.

Bandura, A. (2003). Auto-efficacité, le sentiment d'efficacité personnelle (J. Lecomte, Trans.). Paris, France : DeBoeck.

Bandura, A. (2006). Guide for constructing self-efficacy scalses. Dans F. Pajares et T. Urdan (dir.) Self-Efficacy Beliefs of Adolescents (p. 307-337). Charlotte, NC : Information Age Publishing.

Brown, C. E., Wickline, M. A., Ecoff, L., et Glaser, D. (2009). Nursing practice, knowledge, attitudes and perceived barriers to evidence-based practice at an academic medical center. Journal of Advanced Nursing, 65(2), 371-381. https://doi.org/10.1111/j.13652648.2008.04878.x

Callister, L. C., Matsumura, G., Lookinland, S., Mangum, S., et Loucks, C. (2005). Inquiry in Baccalaureate Nursing Education: Fostering Evidence-Based Practice. Journal of Nursing Education, 44(2), 59-64.

Chouinard, M. C., Lavoie, M., Poitras, M. E., Sasseville, M., De Jordy, C., et Girard, A. (2015). Utilisation d'un club de lecture comme méthode pédagogique pour favoriser l'appropriation des données probantes par des étudiantes au baccalauréat en sciences infirmières. Recherche en soins infirmiers, 120.

Christie, J., Hamill, C., et Power, J. (2012). How can we maximize nursing students' learning about research evidence and utilization in undergraduate, preregistration programmes? A discussion paper. Journal of Advanced Nursing, 68(12), 2789-2801. https://doi.org/10.1111/j.1365-2648.2012.05994.x

DiCenso, A., Guyatt, G., et Ciliska, D. (2005). Evidence-Based Nursing. A Guide to Clinical Practice. St. Louis, MI: Mosby Elsevier.

Faculté des sciences infirmières, Université de Montréal. (2012). Démarche Humaniste-caring. Montréal, QC : Auteur.

Faculté des sciences infirmières Université de Montréal. (2015). Référentiel de compétences, baccalauréat en sciences infirmières. Montréal, QC : Auteur.

Finkelman, A., et Kenner, C. (2009). Teaching IOM: Implications of the Institute of Medicine Reports for Nursing Education ( $2^{\mathrm{e}}$ éd.). Silver Spring, MD : American Nurse Association.

Forsman, H., Wallin, L., Gustavsson, P., et Rudman, A. (2012). Nursing students' intentions to use research as a predictor of use one year post graduation: a prospective study. International Journal of Nursing Studies, 49(9), 1155-1164. https://doi.org/10.1016/j.ijnurstu.2012.04.002 
Foss, J. E., Kvigne, K., Larsson, B. W., et Athlin, E. (2014). A model (CMBP) for collaboration between university college and nursing practice to promote research utilization in student's clinical placements: A pilot study. Nurse Education in Practice, 14, 396-402. https://doi.org/10.1016/j.nepr.2013.11.008

Gray, M. T. (2010). Research odyssey: the evolution of a research partnership between baccalaureate nursing students and practicing nurses. Nurse Education Today, 30(4), 376-382. https://doi.org/10.1016/j.nedt.2009.10.001

Heye, M. L., et Stevens, K. R. (2009). Using New Resources to Teach Evidence-Based Practice. Journal of Nursing Education, 48(6), 334-339. https://doi.org/10.3928/0148483420090515-06

Jakubec, S. L., et Astle, B. (2013). Students Connecting Critical Appraisal to Evidence-Based Practice: A Teaching-Learning Activity for Research Literacy. Journal of Nursing Education, 52(1), 56-58. https://doi.org/10.3928/01484834-20121121-03

Jones, C. J., Crookes, P. A., et Johnson, K. M. (2011). Teaching critical appraisal skills for Nursing research. Nursing Education in Practice, 11, 327-332. https://doi.org/10.1016/j.nepr.2011.03.002

Kajermo, K. N., Bostrom, A. M., Thompson, D. S., Hutchinson, A. M., Estabrooks, C. A., et Wallin, L. (2010). The BARRIERS scale -- the barriers to research utilization scale: A systematic review. Implementation Science, 5(1). https://doi.org/10.1186/1748-5908-5-32

Kameg, K., Howard, V. M., Clochesy, J., Mitchell, A. M., et Suresky, J. M. (2010). The impact of high fidelity human simulation on self-efficacy of communication skills. Issues in Mental Health Nursing, 31(5), 315-323. https://doi.org/10.3109/01612840903420331

Kennedy, E. (2013). The Nursing Competence Self-Efficacy Scale (NSCES): An Instrument Development and Psychometric Assessment Study (Doctor of Philosophy, Dalhousie University, Halifax, NÉ).

Lasnier, F. (2000). Les concepts qui sous-tendent les programmes de formation (par compétences). Réussir la formation par compétence. Montréal, QC : Guérin.

Levin, R. F., Fineout-Overholt, E., Melnyk, B. M., Barnes, M., et Vetter, M. J. (2011). Fostering evidence-based practice to improve nurse and cost outcomes in a community health setting: a pilot test of the advancing research and clinical practice through close collaboration model. Nursing Administration Quarterly, 35(1), 21-33. https://doi.org/10.1097/naq.0b013e31820320ff

Mattila, L. R., Rekola, L., Koponen, L., et Eriksson, E. (2013). Journal club intervention in promoting evidence-based nursing: perceptions of nursing students. Nurse Education in Practice, 13(5), 423-428. https://doi.org/10.1016/j.nepr.2013.01.010

McLaughlin, K., Moutray, M., et Muldoon, O. T. (2008). The role of personality and selfefficacy in the selection and retention of successful nursing students: a longitudinal study. Journal of Advanced Nursing, 61(2), 211-221. https://doi.org/10.1111/j.13652648.2007.04492.x 
Meeker, M. A., Jones, J. M. et Flanagan, N. A. (2008). Teaching undergraduate nursing research from an evidence-based practice perspective. Journal of Nursing Education, 47(8), 376379. https://doi.org/10.3928/01484834-20080801-06

Melnyk, B. M., \& Fineout-Overholt, E. (2010). Evidence-based practice in nursing and health care: A guide to best practice. Philadelphia, PA : Lippincott Williams \& Wilkins.

Melnyk, B. M., Fineout-Overholt, E., Gallagher-Ford, L., et Kaplan, L. (2012). The State of Evidence-Based Practice in US Nurses: Critical Implications for Nurse Leaders and Educators. The Journal of Nursing Administration, 42(9), 410-417. https://doi.org/10.1097/nna.0b013e3182664e0a

Miecznikoski Phillips, R. (2014). Creative Classroom Strategies for Teaching Nursing Research. Nurse Educator, 39(4), 199-201. https://doi.org/10.1097/nne.0000000000000052

Odell, E., et Barta, K. (2011). Teaching evidence-based practice: the Bachelor of Science in nursing essentials at work at the bedside. Journal of Professional Nursing, 27(6), 370 377. https://doi.org/10.1016/j.profnurs.2011.04.007

Pajares, F., \& Urdan, T. (2006). Self-efficacy beliefs of adolescents (adolescence and education). Charlotte, NC : Information Age Publishing.

Polit, D. F., et Beck, C. T. (2006). Essentials of nursing research : methods, appraisal, and utilization ( $6^{\mathrm{e}}$ éd.). Philadelphia, PA : Lippincott Williams et Wilkins.

Pravikoff, D., Tanner, A., et Pierce, S. (2005). Readiness of US nurses for evidence-based practice. American Journal of Nursing, 105(9), 40-52.

Roy, N. (2014). La classe inversée: une pédagogie renversante? Le Tableau, 3(1).

Schlairet, M. C., Green, R., et Benton, M. J. (2014). The Flipped Classroom, Strategies for an Undergraduate Nursing Course. Nurse Educator, 39(6), 321-325. https://doi.org/10.1097/nne.0000000000000096

Smith-Strom, H., et Nortvedt, M. W. (2008). Evaluation of evidence-based methods used to teach nursing students to critically appraise evidence. Journal of Nursing Education, 47(8), 372-375. https://doi.org/10.3928/01484834-20080801-08

Squires, J. E., Estabrooks, C. A., Gustavsson, J. P., et Wallin, L. (2011). Individual determinants of research utilization by nurses: a systematic review update. Implementation Science, 6(1). https://doi.org/10.1186/1748-5908-6-1

Strickland, K., Gray, C., et Hill, G. (2012). The use of podcasts to enhance research-teaching linkages in undergraduate nursing students. Nurse Education in Practice, 12(4), 210-214. https://doi.org/10.1016/j.nepr.2012.01.006

Swenson-Britt, E., \& Reineck, C. (2009). Research education for clinical nurses: a pilot study to determine research self-efficacy in critical care nurses. Journal of Continuing Education in Nursing, 40(10), 454-461. https://doi.org/10.3928/00220124-20090923-05

Tardif, J. (2006). L'évaluation des compétences, documenter le parcours de développement. Montréal, QC : Chenelière Éducation. 
Thomson, P., Smith, A., et Annesley, S. (2014). Exploration of the effects of peer teaching of research on students in an undergraduate nursing programme. Journal of Research in Nursing, 19(5), 415-430. https://doi.org/10.1177/1744987113519444

Wallen, G. R., Mitchell, S. A., Melnyk, B. M., Fineout-Overholt, E., Miller-Davis, C., Yates, J., et Hastings, C. (2010). Implementing evidence-based practice: effectiveness of a structured multifaceted mentorship programme. Journal of Advanced Nursing, 66(12), 2761-2771. https://doi.org/10.1111/j.1365-2648.2010.05442.x

Wallin, L., Bostrom, A. M., et Gustavsson, J. P. (2012). Capability beliefs regarding evidencebased practice are associated with application of EBP and research use: validation of a new measure. Worldviews Evidence Based Nursing, 9(3), 139-148. https://doi.org/10.1111/j.1741-6787.2012.00248.x

Watt, E., Murphy, M., Pascoe, E., Scanlon, A., et Gan, S. (2011). An evaluation of a structured learning programme as a component of the clinical practicum in final year bachelor of nursing programme: a pre-post-test analysis. Journal of Clinical Nursing, 20(15-16), 2286-2293. https://doi.org/10.1111/j.1365-2702.2010.03621.x

Winters, C. A., et Echeverri, R. (2012). Teaching strategies to support evidence-based practice. Critical Care Nurse, 32(3), 49-54. https://doi.org/10.4037/ccn2012159

Zhang, Q., Zeng, T., Chen, Y., \& Li, X. (2012). Assisting undergraduate nursing students to learn evidence-based practice through self-directed learning and workshop strategies during clinical practicum. Nurse Education Today, 32(5), 570-575. https://doi.org/10.1016/j.nedt.2011.05.018 